\title{
Immunité innée \\ Expression cutanée \\ et fonction \\ des récepteurs Toll-like
}

> Les récepteurs Toll-like (TLR) sont des récepteurs membranaires de découverte récente, Philippe Musette, Isabelle Auquit Auckbur, Édouard Begon impliqués dans l'immunité immédiate. Découverts chez la drosophile, ils se sont révélés être des structures phylogénétiquement très conservées. Ubiquitaires dans l'organisme, ils sont particulièrement présents dans les cellules de l'immunité et les tissus barrières. Les TLR, au nombre de 11 chez l'homme, reconnaissent un petit nombre de structures moléculaires propres aux micro-organismes, communes à de nombreux pathogènes. Les TLR peuvent également être activés par des ligands endogènes physiologiques. L'activation d'un TLR est à l'origine d'une cascade signalétique aboutissant à la translocation nucléaire de $\mathrm{NF}-\kappa \mathrm{B}$ et à la sécrétion de cytokines pro-inflammatoires. La peau, qui constitue une barrière contre les aggressions, exprime de nombreux TLR à la surface des kératinocytes. <

\section{Structure}

Les TLR sont des protéines transmembranaires de type 1 , fidèlement conservées au cours de l'évolution $[1,2]$. Sur la base de la similarité de leurs domaines intracytoplasmiques, les TLR sont liés à la famille des récepteurs de l'interleukine-1 (IL-1). Chaque TLR contient un domaine extracellulaire riche en séquences répétées de leucine, participant à la reconnaissance $\mathrm{du}$ ligand, et un domaine intracellulaire contenant une région appelée Toll-IL-IR homology (TIR) domain. Ce domaine TIR est une structure phylogénétiquement conservée, que partagent les TLR, le domaine intracytoplasmique des récepteurs de l'ILI et de I'IL-18, ainsi que la protéine MyD88. Celle-ci est une protéine adaptatrice qui, après interaction avec les TLR par son propre domaine TIR carboxyterminal, active la transduction du signal liée à la fixation du ligand par le TLR (Figure 1). Les gènes codant pour les TLR sont positionnés à différents endroits du génome, mais certains sont

Article reçu le 20 avril 2005, accepté le 24 octobre 2005.

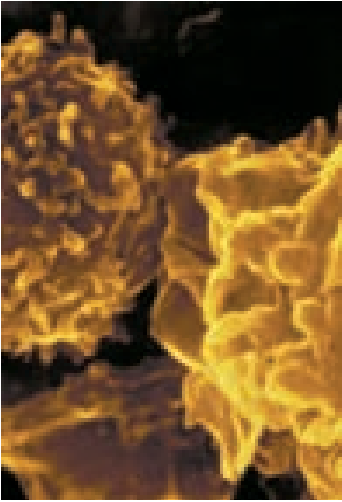

\section{P. Musette :}

Service de dermatologie.

I. Auquit Auckbur:

Service de chirurgie plastique.

Hôpital Charles Nicolle,

1, rue Germont,

76031 Rouen, France.

દ. Begon :

Service de dermatologie,

regroupés (TLR 1, 6, 10 Hôpital Saint-Louis,

sur le chromosome 4, 1, avenue Claude-Vellefaux,

TLR 7 et 8 sur le chromosomeX).

\section{Expression cellulaire et tissulaire}

75010 Paris, France.

Philippe.Musette@chu-rouen.fr hmn.ap-hop-paris.fr

La densité antigénique des TLR à la surface des cellules est faible: quelques milliers de molécules par cellule dans les monocytes, et quelques centaines dans les cellules dendritiques immatures. Bien souvent, l'expression intracytoplasmique est plus importante.

La plupart des tissus expriment au moins un TLR, certains types cellulaires exprimant la totalité du répertoire connu (splénocytes, leucocytes du sang périphérique) [2-5]. Les compartiments de l'organisme engagés dans la réponse immune, ainsi que les tissus les plus exposés à l'environnement microbien (poumon, intestin grêle, côlon), possèdent le plus grand répertoire de TLR. Le répertoire des TLR exprimés par les cellules mononucléées du sang circulant a fait l'objet de nombreuses études par RT-PCR quantitative, montrant que le répertoire Toll est variable selon les cellules engagées dans la réponse immunitaire. Ainsi, les TLRl et 6 sont exprimés dans tous les types cellulaires; les TLR 2, 4 et 8, de leur côté, sont principalement présents dans les monocytes $\mathrm{CD} 14^{+}$, tandis que les TLR9 et 10 sont exprimés à un 
niveau élevé dans les cellules B CD19+. Le TLR3 est, quant à lui, exprimé sélectivement par les cellules dendritiques.

Le répertoire des TLR diffère également au sein d'une même famille cellulaire: les monocytes expriment préférentiellement les TLR1, 2, 4, 5 et 8, tandis que les cellules dendritiques immatures expriment sélectivement le TLR 3, et les cellules dendritiques plasmocytoïdes les TRL7 et 9, à un niveau élevé. La variété et le niveau d'expression des TLR varie en fonction de la maturation du monocyte vers la cellule dendritique: ainsi, l'expression du TLR3 est accrue lors du passage du monocyte vers la cellule dendritique immature, tandis que celle du TLR5 decroît.

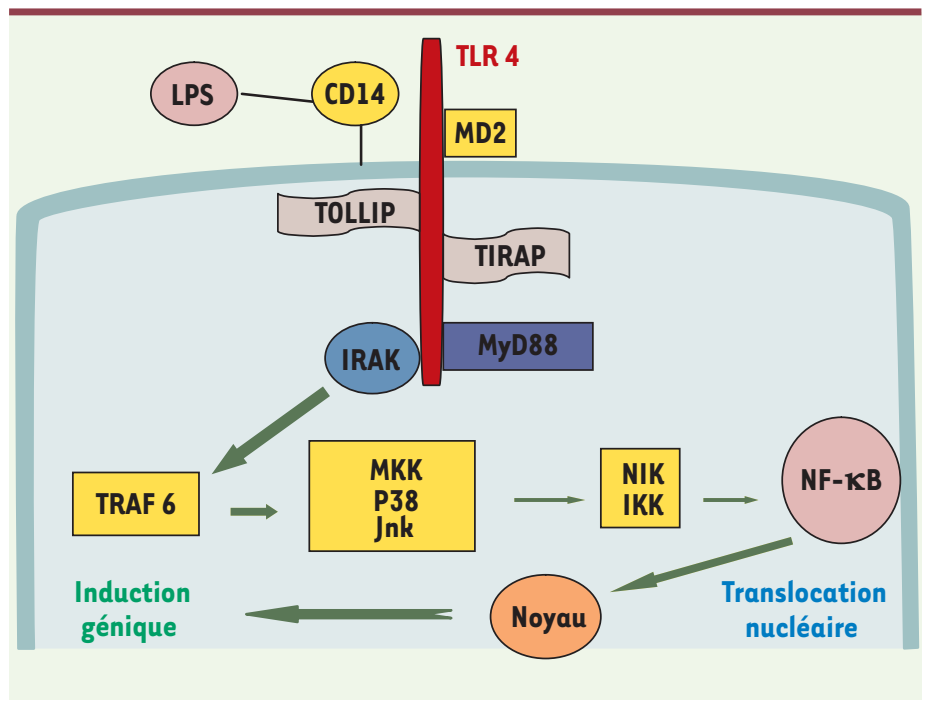

Figure 1. Transduction du signal après fixation du ligand par le TLR : voie NFKB. LPS: lipopolysacharide; TOLLIP: Toll/IL-IR domain-containing adaptor protein; TIRAP: Toll-IL-1 receptor domain-containing adapter protein; MyD88: myeloid differentiation factor 88; IRAK: IL-IR-associated kinase I; MKK : MAPK (mitogen-activated protein kinase) kinase; TRAF $6:$ TNF $\alpha$ receptorassociated factor 6 ; JNK : c-Jun NH2-terminal kinase; NIK: $N F-K B$ inducing kinase; IKK : inhibitor of $k B$ kinase; NF- $K B$ : nuclear factor $\kappa B(\rightarrow)$

$(\rightarrow) \mathrm{m} / \mathrm{s} 2004$, $n^{\circ} 12$, p. 1119
De nombreux ligands infectieux des TLR (TLR 2, 3, 4, 5, 9) ont été mis en évidence (Figure 2): la multiplicité des agonistes possibles, de même que la diversité de structure des différents TLR, étonnantes, n'ont pour l'instant pas reçu d'explication satisfaisante. D'autres TLR ne semblent pas avoir de ligand spécifique: ainsi, les TLR 1, 6 et 10 seraient des corécepteurs, agissant en association avec les autres TLR en modulant leur réponse. Le rôle des TLR 8 et 10 est, quant à lui, encore à préciser.

Le TLR2 est celui qui reconnaît le plus grand nombre de molécules associées aux pathogènes; il reconnaît en effet de nombreuses structures moléculaires qui sont des composants communs de la paroi de différentes familles de bactéries: lipoprotéines, acides lipotochéiques, peptidoglycane, lipo-arabinomannane. Le TLR2 est ainsi le médiateur de la réponse cellulaire à des bactéries appartenant à différentes familles, ainsi qu'à des agents fongiques: Borrelia burgdorferi, Mycobacterim avium, Mycobacterium tuberculosis, streptocoques du groupe B, Mycoplasma fermentans, Staphylococcus aureus ou, encore, Treponema pallidum. Si le LPS, principal composant des bactéries Gram-, est reconnu spécifiquement par le TLR4, le LPS d'autres bactéries non entérales a pour médiateur le TLR2 (Porphyromonas gingivalis, Leptospira). On peut noter que le polymorphisme humain du TLR2 est associé à une susceptibilité accrue aux infections staphylocociques et méningococciques.

Le TLR3 a pour ligand I'ARN double brin, une structure moléculaire associée aux infections virales, produite par de nombreux virus durant leur étape de réplication. Quant au TLR4, il a pour principal ligand le lipopolysaccharide des bactéries Gram-. La délétion spontanée ou induite du gène du TLR4 chez la souris induit ainsi une absence de réponse au LPS et une résistance au choc septique. De son côté, le polymorphisme humain de TLR4 est associé à une diminution de la réponse au LPS. D'autres ligands microbiens du TLR4 ont également été découverts: la protéine de fusion du virus respiratoire synticial, Haemophilus influenzae et Candida albicans.

Le TLR5 a pour ligand la flagelline des bactéries gram + et -, une protéine pro-inflammatoire néces-

\section{Ligands exogènes}

Les TLR reconnaissent des structures moléculaires associées aux pathogènes, les pathogens associated molecular patterns (PAMP) [2-5]. Ces molécules, spécifiques des bactéries, sont très conservées, et donc retrouvées dans de nombreuses familles bactériennes. La stratégie de l'immunite innée n'est donc pas de reconnaître tous les antigènes possibles, mais un petit nombre d'entre eux, sous la forme de structures hautement conservées présentes dans un important groupe de micro-organismes. saire à la propulsion bactérienne. Elle pourrait jouer un rôle dans la résistance aux infections à salmonelles. Le TLR7 ne possède pas de ligand défini ; toutefois, un composant antiviral, l'imidazoquinoline (Imiquimod ${ }^{\circledR}$ ), a récemment été proposé comme ligand de TLR7. De fait, les souris $T L R 7^{-/-}$ne répondent pas à l'imidazoquinoline, qu'il s'agisse de la transduction du signal ou de la maturation des cellules dendritiques.

Enfin, le TLR 9 reconnaît des structures présentes dans l'ADN des mycobactéries, les CpG non méthylés, connus pour leur capacité stimulatrice du système immunitaire (c'est la forme méthylée qui prédomine chez les mamifères). Les souris $T L R 9^{-/-}$sont ainsi incapables 
de produire une réponse inflammatoire après stimulation par les CpG non méthylés, qu'il s'agisse de production de cytokines macrophagiques, de maturation des cellules dendritiques ou de prolifération des cellules $B$.

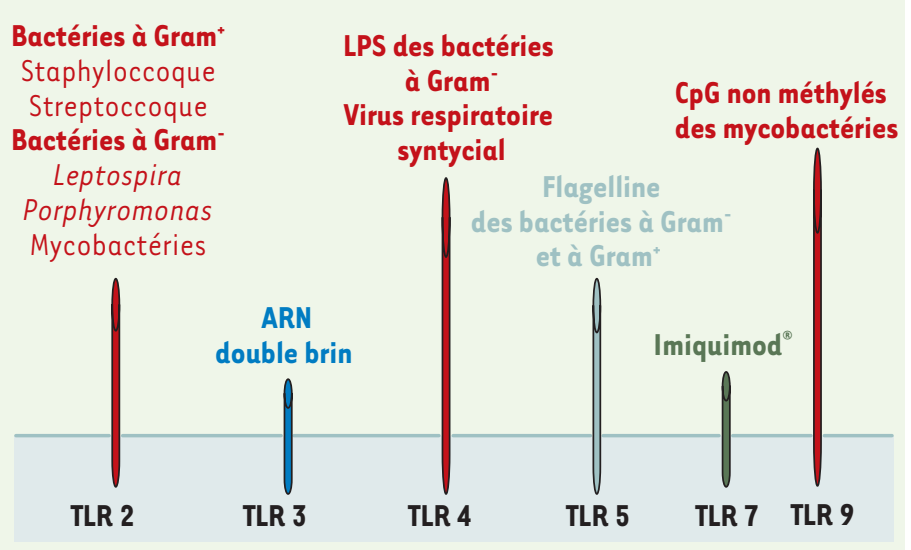

Figure 2. Ligands infectieux des TLR.

\section{Ligands endogènes physiologiques}

Plusieurs études ont mis en évidence que des signaux de dommage interne, en plus des molécules associées aux pathogènes, étaient à l'origine d'une réponse inflammatoire impliquant les TLR (Figure 3). Des molécules variées sont libérées par des cellules subissant des dommages irrémédiables, notamment dans des situations d'ischémie ou d'inflammation. Au contraire d'une mort cellulaire par apoptose, ces dommages peuvent modifier la structure des protéines exprimées à la surface cellulaire, ou permettre la translocation de protéines normalement non exprimées à la membrane. Durant la réponse inflammatoire, la production et la dégradation de protéines variées de la matrice extracellulaire sont également accrues : ces composants dégradés sont à l'origine de réactions inflammatoires dont certaines impliquent les TLR 2, 3, 4 et 5.

Le TLR4 reconnaît ainsi plusieurs ligands physiologiques tels que la protéine végétale Taxol, la protéine de choc thermique HSP 60 , le fibrinogène, les oligosaccharides de hyaluronane ou encore le protéoglycane d'héparane sulfate. Le peroxyde d'hydrogène, molécule produite par les myocytes cardiaques en situation d'ischémie, active la voie NF- $\kappa B$ dans des fibroblastes de hamster surexprimant le TLR2, un effet aboli par un anticorps antagoniste de TLR2. Enfin, le TLR9 reconnaît les motifs CpG non méthylés apparaissant chez l'homme au cours de certaines maladies auto-immunes, notamment, dont le lupus et la polyarthrite rhumatoïde.

\section{Expression cutanée}

Les kératinocytes humains exprime un vaste répertoire de TLR [6-8], notamment ceux dont les ligands infectieux sont les plus nombreux et les mieux connus (TLR 2, 34 et 5$)$. Ces résultats ont été obtenus par différentes techniques (immunocytochimie, cytométrie de flux, RT-PCR, Western blot) appliquées à l'analyse de plusieurs souches de kératinocytes normaux, issus de différents patients, et de lignées de kératinocytes immortalisées [6]. L'existence d'un répertoire de TLR important dans la peau est en accord avec les connaissances acquises sur leur distribution dans les différents compartiments de l'organisme: en effet, il a été montré que les tissus barrières, les plus exposés à l'agression microbienne, sont dotés d'un répertoire de récepteurs plus varié que les autres tissus. La peau, comme les épithéliums intestinaux et bronchiques, apparaît ainsi comme un tissu «frontière » (toll) dans lequel les informations captées par l'immunité immédiate sur son environnement microbien est rapidement transmise aux agents de l'immunité innée et adaptative.

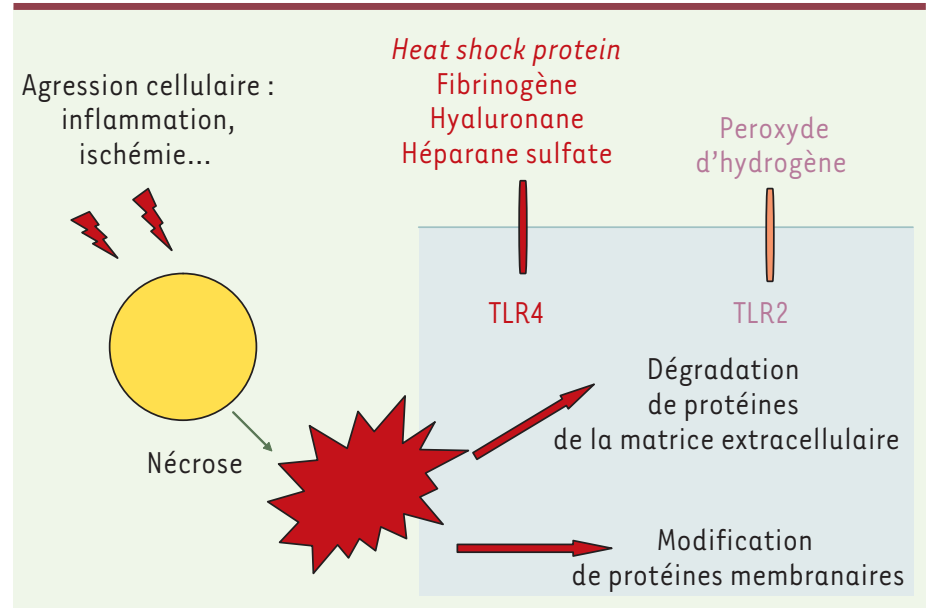

Figure 3. Ligands endogènes physiologiques des TLR. Des situations telles que I'ischémie ou l'inflammation peuvent déclencher une réponse inflammatoire impliquant les TLR soit en raison d'une modification de la nature ou de la structure des protéines de surface, soit parce que la production et la dégradation de protéines de la matrice extracellulaire sont accrues.

\section{Un rôle pour les TLR dans le psoriasis?}

Le psoriasis est une dermatose inflammatoire chronique, essentiellement caractérisée par une prolifération kéra- $(\rightarrow) \mathrm{m} / \mathrm{s} 2006$, $n^{\circ} 2$, p. 164 tinocytaire et par l'infiltration dermique de lymphocytes $T$ et de cellules inflammatoires $(\rightarrow)$.

Les événements déclenchant cette réponse immune $T$ sont encore inconnus. 
Nous avons toutefois montré l'existence d'une surexpression du TLR2 en cas de psoriasis, associée à une expression de TLR4 comparable à celle de témoins [6]. Par ailleurs, I'IFN $\gamma$, le TNF $\alpha$ (tumor necrosis factor $\alpha$ ) et I'IL-8, cytokines-clés abondamment présentes dans la peau psoriasique, sont également produites après stimulation des TLR2 et TLR4 sur les kératinocytes [6-9]: il est probable que des ligands endogènes (protéines de choc thermique) ou exogènes (pepdidoglycanes issus de streptocoques) pourraient induire, via les récepteurs TLR2 et TLR4, la libération de cytokines pro-inflammatoires au cours du psoriasis. Enfin, l'application d'un ligand de TLR7 sur la peau peut, dans certaines conditions, induire des lésions de psoriasis [10].

\section{Conclusions}

Il apparaît donc que les TLR2, 3 et 4 exprimés par les kératinocytes sont fonctionnels et peuvent induire la libération de TNF $\alpha$ et d'IL-8. Les TLR pourraient donc jouer un rôle dans l'inflammation cutanée aussi bien au cours de la défense anti-infectieuse que dans des maladies cutanées inflammatoires comme l'eczéma ou le psoriasis. $\diamond$

\section{SUMMARY}

Cutaneous expression of Toll-like receptors

Toll receptors were first identified as an essential molecule for embryonic patterning in Drosophila and were subsequently shown to be a key in antibacterial and antifungal immunity in adult flies. Toll receptors have been conserved throughout evolution. In mammals, TLRs have been implicated in both inflammatory responses and innate host defense to pathogens. The 11 different TLRs recognize conserved molecular patterns of micro- bial pathogens termed pathogen-specific molecular patterns (PAMPs), that permit to confer responsiveness to a wide variety of pathogens. Endogenous ligands are also able to activate TLRs. All adult tissue is capable to express at least one of member of TLR family, but a largest repertoire of TLRs is found in tissues exposed to the external environment. The TLR activation induce the NF- $\kappa B$ translocation to the nucleus and cytokine secretion. Since the primary function of skin is to provide an effective barrier against outside agression, it is likely that keratinocytes may play a role in a rapid and efficient host defence system, and the fact that keratinocytes are capable of expressing a wide variety of TLRs is subsequently not surprising. $\diamond$

\section{RÉFÉRENCES}

1. Medzhitov R. Toll-like receptors and innate immunity. Nat Rev Immunol 2001 ; 1 : 135-45.

2. Akira S. Toll-like receptors: critical proteins linking innate and acquired immunity. Nat Immunol $2001 ; 2: 675-80$.

3. Lemaitre B, Nicolas $\varepsilon$, Michaut L, et al. The dorsoventral regulatory gene cassette spatzle/ toll/cactus control the potent antifungal response in Drosophila adults. Cell 1996 ; $86: 973-83$.

4. Medzhitov R. Innate immunity. N Engl J Med 2000 ; 343 : 338-44.

5. Fearon DT. The instructive role of innate immunity in the acquired immune response. Science $1996 ; 272: 50-3$.

6. Begon $\varepsilon$, et al. A large repertoire of TLR is expressed on normal keratinocyte: up-regulation of TLR2 in psoriasis. 2006 (soumis pour publication).

7. Pivarcsi A, Bodai L, Rethi B, et al. Expression and function of Toll-like receptors 2 and 4 in human keratinocytes. Int Immunol $2003 ; 15: 721-30$.

8. Mempel M, Voelcker V, Kollisch G, et al. Toll-like receptor expression in human keratinocytes nuclear factor kappaB controlled gene activation by staphylococcus aureus is toll-like receptor 2 but not toll-like receptor 4 or platelet activating factor receptor dependent. J Invest Dermatol 2003 ; 121 : 1389-96.

9. Baker BS, Ovigne JM, Powles AV, Corcoran S, Fry L. Normal keratinocytes express Toll-like receptors (TLRs) 1, 2, 5: modulation of TLR expression in chronic plaque psoriasis. BrJ Dermatol $2003 ; 148: 670-9$.

10. Gilliet M, Conrad C, Geiges M, et al. Psoriasis triggered by toll-like receptor 7 agonist imiquimod in the presence of dermal plasmocytoid dendritic cell precursors. Arch Dermatol $2004 ; 140: 1490-5$.

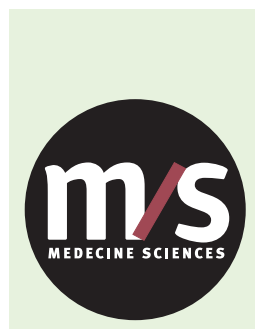

Tarifs d'abonnement M/S - 2006

Abonnez-vous

à Médecine/Sciences
> Depuis 20 ans, grâce à $m / s$, vous vivez en direct les progrès des sciences biologiques et médicales

TIRÉS À PART

P. Musette 